\title{
Relative area and relative luminance combine to anchor surface lightness values
}

\author{
XIAOJUN LI and ALAN L. GILCHRIST \\ Rutgers University, Newark, New Jersey
}

\begin{abstract}
The anchoring of lightness perception was tested in simple visual fields composed of only two regions by placing observers inside opaque acrylic hemispheres. Both side-by-side and center/surround configurations were tested. The results, which undermine Gilchrist and Bonato's (1995) recent claim that surrounds tend to appear white, indicate that anchoring involves both relative luminance and relative area. As long as the area of the darker region is equal to or smaller than the area of the lighter region, relative area plays no role in anchoring. Only relative luminance controls anchoring: The lighter region appears white, and the darker region is perceived relative to that value. When the area of the darker region becomes greater than that of the lighter region, relative area begins to play a role. As the darker region becomes larger and relative area shifts from the lighter region to the darker region, the appearance of the darker region moves toward white and the appearance of lighter region moves toward luminosity. This hitherto unrecognized rule is consistent with almost all of the many previous reports of area effects in lightness and brightness. This in turn suggests that a wide range of earlier work on area effects in brightness induction, lightness contrast, lightness assimilation, and luminosity perception can be understood in terms of a few simple rules of anchoring.
\end{abstract}

Gilchrist and Bonato (1995) recently reported experiments designed to identify the anchoring rule for perceived lightness under the minimal conditions that support the perception of a surface.

An anchoring rule describes how the visual system assigns specific lightness values to surfaces represented in an image. All modern theories of lightness perception hold that, in one way or another, surface lightness is based on relative, not absolute, luminance (intensity). But, without an anchoring rule, relative luminance can specify only relative surface lightness, not absolute or specific lightness values. A rule is needed to provide at least one point of contact between luminance values in the stimulus and values on the black/white surface color scale, as illustrated in Figure 1. This problem has been neglected by most theories (Adelson \& Pentland, 1990; Arend, 1994; Bergström, 1977; Cornsweet, 1970; Gilchrist, 1979; Heinemann, 1972; Jameson \& Hurvich, 1964), although several have suggested a rule. Helson (1964) proposed that the average luminance in an image is perceived as middle gray, with the lightness value of other surfaces determined relative to this standard, or anchor. This is closely related to what has been called, mainly in the chromatic domain, the gray world assumption (Brown, 1994; Buchsbaum, 1980;

The authors wish to acknowledge the support of the National Science Foundation (Grants DBS-9222104 and SBR 95-14679) and to thank Hal Sedgwick, Sten Sture Bergström, Walter Gerbino, and an anonymous reviewer for their constructive comments. Correspondence should be addressed to A. L. Gilchrist, Psychology Department, Rutgers University, Newark, NJ 07102 (e-mail: alan@psychology.rutgers.edu).

-Accepted by previous editor, Myron L. Braunstein
Hurlbert, 1986). Wallach (1948) and others (Horn, 1974; Land \& McCann, 1971) proposed that the highest luminance in a scene is perceived as white, with this value serving as the standard for other surfaces.

Empirical results have favored the highest luminance rule over the average luminance rule in those few tests that have been done (Bruno, 1992; Bruno, Bernardis, \& Schirillo, 1997; Cataliotti \& Gilchrist, 1995; Schirillo \& Shevell, 1993; Wallach, 1948). But Gilchrist and Bonato (1995) noted a serious challenge to the highest luminance rule: the perception of regions that appear selfluminous. According to the highest luminance rule, white is a ceiling. Surfaces brighter than white-that is, selfluminous-should not appear, according to this rule. Yet even Wallach $(1976$, p. 8$)$ observed that when the luminance of the disk becomes greater than that of the annulus, the disk begins to appear luminous.

Gilchrist and Bonato (1995) conducted a set of experiments to resolve this dilemma. They examined anchoring under two basic stimulus conditions: (1) a disk embedded in a Ganzfeld, and (2) a disk/annulus display surrounded by darkness. The simplest image that can produce the experience of a surface contains only a single edge (Gelb, 1929; Katz, 1935), a condition met by the disk/Ganzfeld display but not the disk/annulus display. The disk/annulus configuration was included for historical continuity, but the results did not reveal a clear anchoring rule. Gilchrist and Bonato attributed this to the relative complexity of the disk/annulus/darkness configuration. The disk/Ganzfeld condition produced a clear pattern of results.

On the basis of their findings, Gilchrist and Bonato proposed a new anchoring rule: a surround rule. According to this rule (at least under such minimal conditions), 


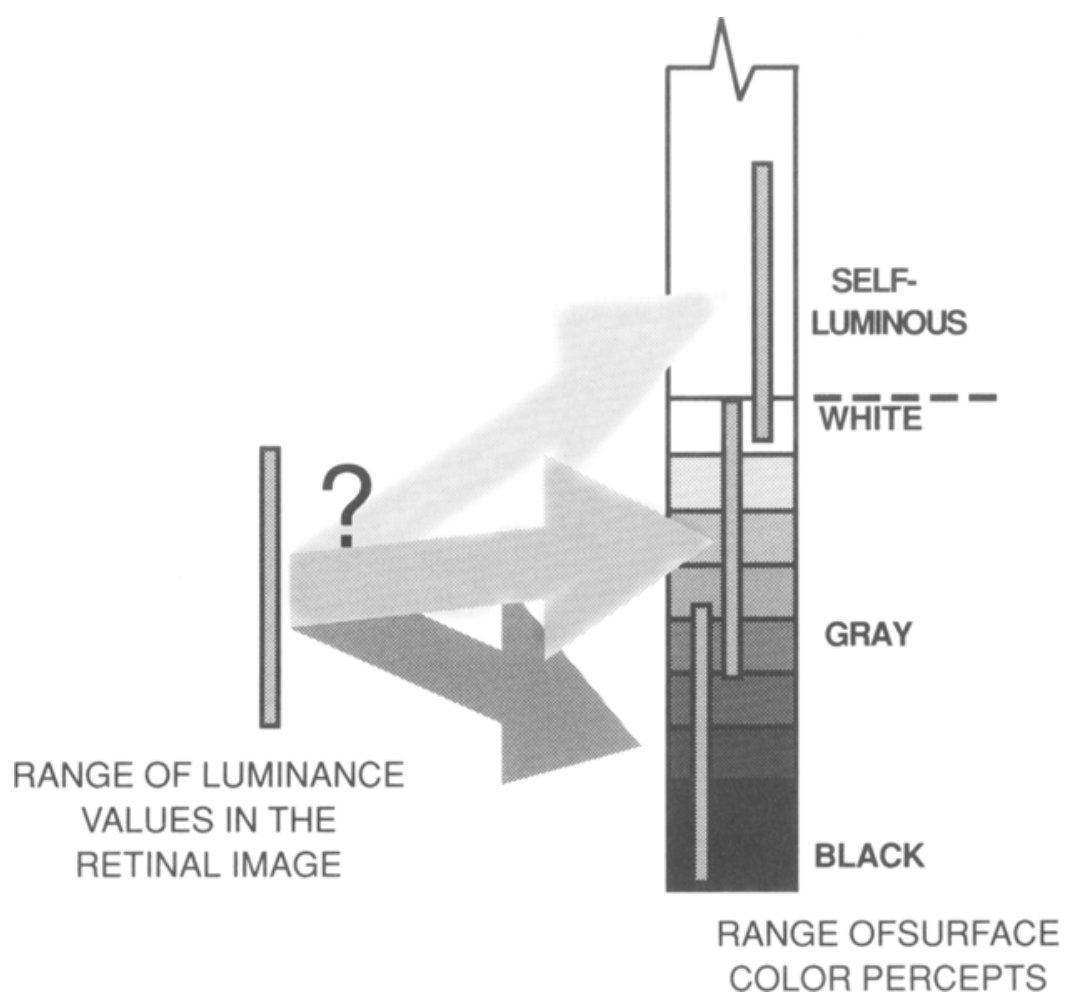

Figure 1. The anchoring problem.

whenever one part of the field surrounds another part, the surrounding region appears white and the lightness of the surrounded region is seen relative to the white surround. When neither of two regions surrounds the other, as would be the case in a Ganzfeld split into two halves, the highest luminance rule presumably governs the outcome. But when one of the two regions surrounds the other, as in the disk/Ganzfeld, the surround rule emerges clearly. The results reported by Gilchrist and Bonato formed an elegant pattern consistent with the surround rule. When the disk was darker than the Ganzfeld, it appeared as some shade of opaque gray. When it was brighter than the Ganzfeld, the disk appeared luminous. In both cases, the Ganzfeld always appeared as an unchanging white.

Gilchrist and Bonato did not resolve the question of whether the Ganzfeld appeared as a constant white in their experiments because it was the surrounding region or because of its large area. In their split Ganzfeld display, the two parts of the visual field had equal areas. But in their disk/Ganzfeld display, the area of the Ganzfeld surround was much greater than the area of the disk.

We now report experiments that seem to resolve the issue of surroundedness versus area. And, to our surprise, it appears to be area, or field size (Katz, 1935), not surroundedness per se, that is the crucial variable. We have used both split Ganzfeld displays and disk/Ganzfeld dis- plays, but, in both kinds of display, we have varied the relative areas of the two regions.

\section{EXPERIMENT 1}

Experiment 1 was designed to test the role of area in a configuration bearing no figure-surround relationship. We used the split Ganzfeld configuration and varied the eccentricity of the vertical edge dividing the two halves of the field. Two displays were tested. In the first display, called the evenly split condition, the vertical edge was centered in the Ganzfeld. In the second display, called the unevenly split condition, the vertical edge was moved to the right of center as far as possible while still keeping it within the visual field for all observers. In both cases, the two regions were painted black and middle gray, respectively. In the unevenly split condition, the larger region was black and the smaller region was middle gray.

\section{Method}

Apparatus. The stimulus was a simple opaque pattern painted onto the inside of a large acrylic hemisphere (henceforth referred to as the dome), $76 \mathrm{~cm}$ in diameter. The observer viewed the pattern by reclining in a comfortable chair, as shown in Figure 2 . The distance from the observer's eye to the center of the dome was approximately $28 \mathrm{~cm}$. The dome was joined to the back of the chair by a large hinge, facilitating convenient entry and exit. A $150-\mathrm{W}$ quartz halogen light was mounted, facing down, on each side of the chair. 


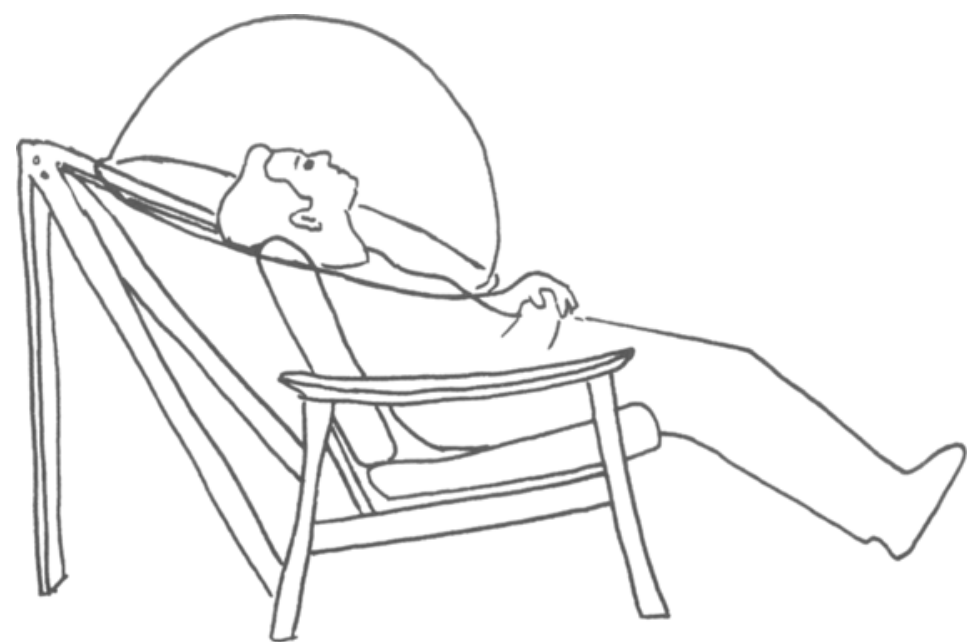

Figure 2. Apparatus used for presenting stimuli.

The floor was covered with white paper so that diffuse light reflecting from the floor illuminated the inside of the dome. Two skirts of black paper were attached to the perimeter of the dome on both the left side and the right side in order to prevent the partial visibility of bright regions in the extreme periphery of the visual field, especially when lateral eye movements were not sufficiently well prevented.

A schematic of the two conditions is shown in Figure 3. In both conditions, the left side was painted black (approximate Munsell value, 2.5 ; reflectance, $4.6 \%$; luminance, $1.92 \mathrm{~cd} / \mathrm{m}^{2}$ ), and the right side was painted middle gray (approximate Munsell value, 5.5 ; reflectance, $25 \%$; luminance, $10.6 \mathrm{~cd} / \mathrm{m}^{2}$ ). In the unevenly split condition, the vertical boundary was $59^{\circ}$ of visual angle from the center. The resulting surfaces were not perfectly homogeneous; some microtexture was visible.

Lightness matches were made using a separately located Munsell chart housed in a wooden box open on the front side. The chart was

\section{PHYSICAL STIMULUS}

\section{APPEARANCE}

\section{(Munsell Values)}
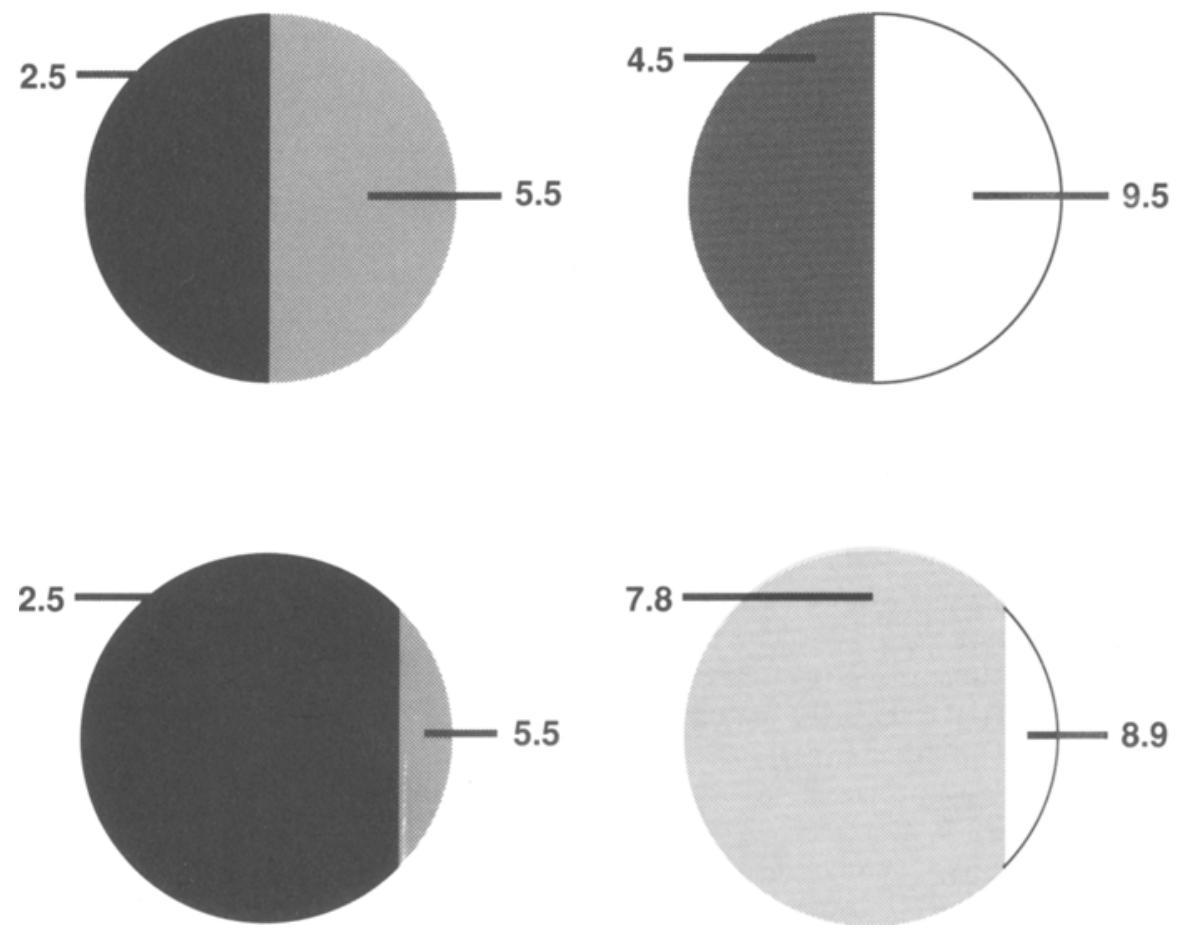

Figure 3. Schematic of the physical stimuli and perceived lightness values in Experiment 1. 
composed of 16 Munsell chips $(1 \times 3 \mathrm{~cm})$, ranging from 2.0 to 9.5 , mounted on white cardboard. It was illuminated by a 15 -W fluorescent bulb, producing a luminance value of $538 \mathrm{~cd} / \mathrm{m}^{2}$ on the $9.5 \mathrm{chip}$.

Procedure. The observer was given a general orientation to the experiment and then, with eyes closed, was led into the laboratory and seated in the apparatus. The dome was lowered into place, the lights were turned on, and the observer was asked to open his or her eyes and to fixate straight ahead. The vertical edge was used for fixation in the evenly split condition and a slight irregularity in the surface was used in the unevenly split condition. The observer was asked for a verbal description of the display, including surface colors and luminosity. The term luminous region was defined as "a region that looks brighter than white. It is not opaque, and light comes from itself." Then, with the observer's eyes closed, the dome was opened, the observer stood up, the dome was closed again, and the observer, with eyes open, was led to the nearby matching chamber. The observer was asked to select, from memory, the two Munsell chips that matched most closely the two shades of gray seen in the dome. In previous work using stimuli that do not fill the entire visual field, we have found that matches made from immediate memory, following the removal of the stimulus, are essentially the same as those made with a Munsell chart in the visual field simultaneously with the stimulus.

Observers and Design. One group of 10 observers served in the unevenly split condition, and a separate group of 10 served in the evenly split condition. There was only a single trial for each observer. The observers were students with a median age of 19 years, almost equally divided between male and female, with normal or corrected-to-normal vision. They volunteered to complete a class requirement and were naive as to the purpose of the experiment.

\section{Results and Discussion}

In both conditions, the middle gray region was perceived as completely white, as indicated in Figure 3, with no significant difference between the two Munsell values of 9.45 and 8.9. This supports the highest luminance rule. In the evenly split condition, the black region was seen as Munsell 4.5 (middle gray), whereas, in the unevenly split condition, it was seen as Munsell 7.8 (very light gray), significantly lighter $(t=2.2, p<.05)$. This shows a pronounced effect of area, even in the absence of a figure-surround relationship. It implies that the Ganzfeld surround in the Gilchrist and Bonato (1995) experiments may have appeared as a constant white more because of its large visual area than because it was a surrounding region. This in turn suggests that large area is associated with the perception of a white surface, just as is high luminance.

One observer reported the lighter region to appear selfluminous in the evenly split condition; 3 observers reported luminosity for the lighter (and smaller) region in the unevenly split condition. Whenever self-luminosity was reported, we used the highest Munsell value on our chart, 9.5, in our computation of average Munsell value. This was generally acceptable to our observers as well.

The fact that the illumination level in the matching apparatus was about 150 times higher than that in the dome constitutes a minor methodological weakness, because lightness can be contaminated, to some degree, by brightness. But this discrepancy cannot explain the perceptual lightening (perceived vs. actual) that we obtained, because brightness contamination would have the opposite effect on the data. Either the chips would appear too light or the dome surfaces would appear too dark. Either way, the observer would then select darker chips.

Note that, in reporting the results, we can speak of the perceived lightness of a surface relative to its physical reflectance and we can speak of the range between two surfaces relative to the physical range between them. We use the term anchoring for the first of these and the term scaling for the second. Anchoring concerns how to locate a set of luminance values on the scale of perceived lightness that extends from black to white. One can imagine the set of relative luminance values being slid up or down the lightness scale while the relationships among the various luminance values are preserved. Scaling concerns the degree to which the spread of lightness values is greater than (we will call this expansion) or less than (we will call this compression) the spread of the luminance values, without regard to where the set is anchored. Brown and MacLeod (1992) use the terms gamut expansion and gamut compression for the same concepts. The default assumption is that the range of perceived lightness values is commensurate with the range of stimulus luminance values. This is known as the ratio principle.

Of course, any measure of expansion or compression will depend on the scale used for the comparison of stimulus and perceived values. We believe that the proper scale for such a comparison is one of log reflectance (or $\log$ luminance).

This problem would be simpler if there were no anchoring effects occurring at the same time as the scaling effects. For example, if the lighter half of the dome were perceived correctly, we could easily measure any expansion or compression simply by noting whether the darker half was perceived as lighter than, darker than, or equal to its physical value. The choice of scale used would become an issue only if we wished to quantify the expansion or compression. But, in our experiment, the lighter half of the dome (physically middle gray) was perceived as white. Given this, what perceived value in the darker half would represent veridical scaling - that is, what value should we expect assuming neither expansion or contraction?

We argue that veridical scaling occurs when the luminance ratio (reflectance ratio, or log difference) between the two matching reflectances is equal to the luminance ratio between the two physical reflectances. This is consistent with Weber's law and with Wallach's (1948) finding that equal ratios produce equal perceived differences in lightness. The Munsell scale does not seem to be an appropriate candidate for such a scale. The Munsell scale was designed to provide equal psychological steps under certain standard conditions. Our conditions were not at all similar to those standard conditions. Furthermore, a scale that provides equal psychological steps does not seem to be an appropriate measure of the physical stimulus.

On the basis of the definition of veridical scaling given above, we can test for distortions in the range of perceived gray shades by comparing the physical reflectance ratio between the two halves of the dome, with the the reflec- 
tance ratio between the mean matched reflectance values for the two halves. In the evenly split condition, the matching lightness values showed a reflectance range (5.7:1) that was slightly expanded relative to the physical reflectance values (5.3:1), in addition to the fact that they were shifted upward on the lightness scale due to anchoring. But, in the unevenly split condition, the perceived lightness range of 1.4:1 was markedly compressed relative to the actual 5.3:1 range.

\section{EXPERIMENT 2}

In Experiment 2, the effect of relative area was tested in the presence of a figure-surround relationship. In one condition, a small disk was surrounded by a Ganzfeld background, using area proportions similar to those in Gilchrist and Bonato's (1995) disk/Ganzfeld experiments. ${ }^{1}$ In a second condition, the disk was enlarged as much as possible for its entire perimeter to remain clearly within the visual field as long as approximate fixation was maintained. This produced an oval-shaped region due to the fact that the visual field is larger horizontally than vertically.

\section{Method}

The method used was identical to that used in Experiment 1 except for the following changes: (1) The stimulus consisted of either a small middle gray disk, $1.35^{\circ}$ of visual angle in diameter, placed in the center of a black Ganzfeld, or a large middle gray oval, $118^{\circ}$ horizontally and $91^{\circ}$ vertically, placed in the center of a black Ganzfeld. The reflectances and luminances of the black and middle gray regions were the same as in Experiment 1. A schematic of the stimuli is shown in Figure 4. (2) In the small disk condition, the observer was instructed to fixate on the disk, whereas, in the large oval condition, the observer was instructed to fixate in the center of the oval so as to keep the entire border of the oval within the visual field. Ten observers viewed each display.

\section{Results and Discussion}

As can be seen in Figure 4, both the small disk and the large oval were seen as white, consistent with the highest luminance rule. The surround did not appear white in either of these conditions, a clear violation of the surround rule. The Ganzfeld surround was matched to a Munsell 6.9 in the small disk condition and a 6.0 in the large oval condition. This difference was not quite significant $[t(18)=1.9, p>.05]$, although the direction of this difference was consistent with the principle that a larger area is associated with a whiter appearance.

It is somewhat puzzling that the Ganzfeld background in the small disk condition did not appear lighter than 6.9. In the most similar disk/Ganzfeld condition tested by Gilchrist and Bonato (1995), the Ganzfeld surround appeared completely white (9.5). Also, $60 \%$ of their ob-

\section{PHYSICAL STIMULUS}

\section{APPEARANCE}

\section{(Munsell Values)}
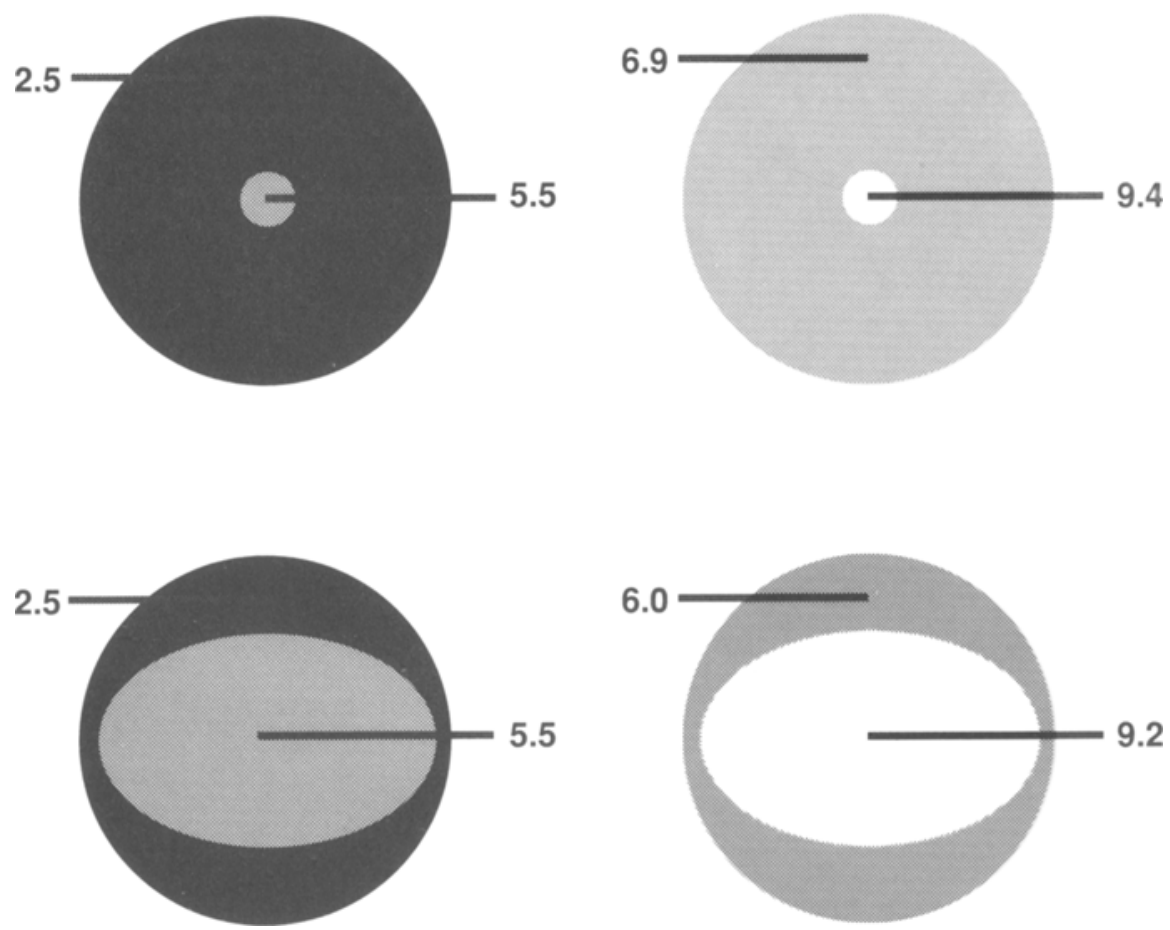

Figure 4. Schematic of the physical stimuli and perceived lightness values in Experiment 2. 
servers reported the disk to be luminous, whereas we obtained no reports of luminosity for the disk. In a pilot study testing the same configuration but using a larger dome, we obtained mean Munsell matches of 8.0 for the Ganzfeld background and 9.5 for the small disk, with 5 observers out of 10 reporting luminosity in the disk. When we replaced the gray disk with one slightly darker than the black background, we obtained a match of 8.9 for the Ganzfeld background (with no reports of luminosity, not surprisingly).

Despite several methodological differences among these three studies, there is no obvious factor that can account for the discrepant results. ${ }^{2}$ We are inclined to believe that the 6.9 value we obtained for the Ganzfeld background is, for whatever reason, somewhat darker than what will normally be obtained for the small disk condition. This expectation is consistent with our general finding of a lightened appearance in the darker region as its visual area increases.

For both the small disk and the large oval conditions, that range of the matched reflectance values was substantially compressed relative to the stimulus range. The actual reflectance range of 5.3:1 produced perceived ranges of 2.1:1 in the small disk condition and 2.8:1 in the large oval condition. The only report of luminosity came from a single observer, who reported it in the large oval condition.

\section{EXPERIMENT 3}

In Experiment 3, we tested decremental centers in the disk/Ganzfeld configuration.

\section{Method}

The method used in Experiment 3 was identical to that of Experiment 2 with the sole exception that now the small disk or large oval was black while the Ganzfeld surround was middle gray. A schematic of the displays is shown in Figure 5.

\section{Results and Discussion}

As shown in Figure 5, in both conditions, the Ganzfeld surround was perceived as completely white. This result is consistent with the highest luminance rule, the surround rule, and the area effect we have been discussing. But the mean Munsell match made to the small disk was 4.2 , whereas the mean Munsell match made to the oval was 6.2. This difference was significant $[t(18)=5.0, p<$ $.0001]$. Here, again, as in Experiment 1, we saw a substantial lightening effect of area. One observer reported

\section{PHYSICAL STIMULUS}

\section{APPEARANCE}

\section{(Munsell Values)}
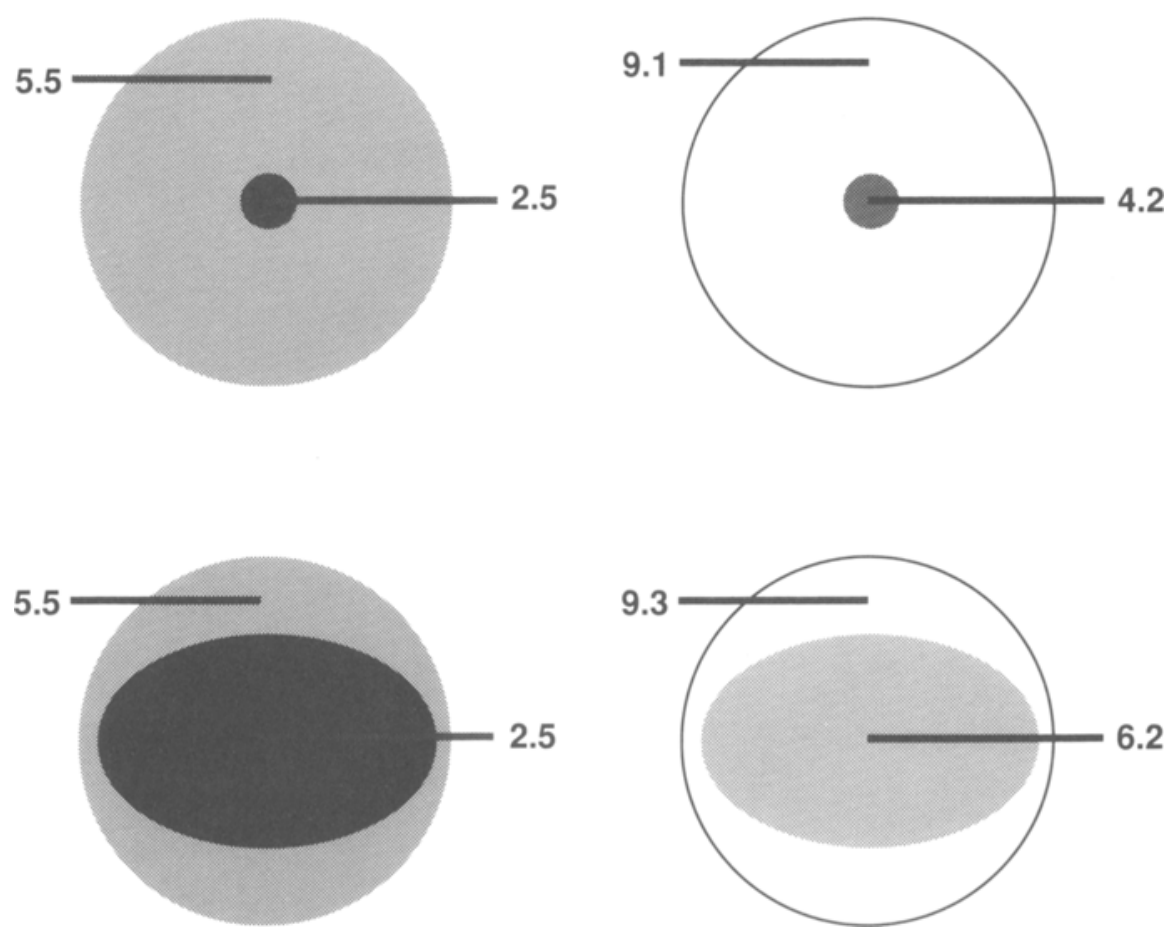

Figure 5. Schematic of the physical stimuli and perceived lightness values in Experiment 3. 
luminosity for the Ganzfeld background in the small disk condition.

In the small disk condition, the perceived reflectance range was 6.1:1. This represents a modest expansion relative to the 5.3:1 physical range. In the large oval condition, the perceived reflectance range was $2.6: 1$, representing a substantial compression, as found in the unevenly split condition of Experiment 1 . Again, the compression seems to be associated with a conflict between highest luminance contribution to anchoring and the largest area contribution. The compression occurred only when the region with the lesser luminance had the greater area. The region with the highest luminance appeared white, whereas the darker region, because of its enlarged area, seems to have been pushed along the lightness scale toward white. As a result, the difference (on the scale of $\log$ reflectance) between the perceived reflectance values of the two regions was squeezed, becoming substantially less than the difference between their actual reflectance values.

\section{GENERAL DISCUSSION}

Our results, taken as a whole, support the general claim made by Gilchrist and Bonato (1995) that geometric factors play an important role in anchoring in addition to photometric factors. But the surround rule, as proposed by Gilchrist and Bonato, does not seem to be correct. That surrounds do not always appear white becomes most obvious in the large oval experiment of Experiment 2. The surround was perceived as light middle gray (Munsell 6.0), not white.

The comparison between the large oval condition of Experiment 3 and the large oval condition of Experiment 2 is particularly relevant to the surround rule hypothesis. The only difference between these two conditions was that the lighter and darker regions were reversed, in terms of which region surrounded and which was surrounded. According to the surround rule, the darker region should have appeared ideally white, but at least lighter in Experiment 2, where it was the surrounding region, than in Experiment 3, where it was the surrounded region. But, in fact, it appeared as a Munsell 6.0 in Experiment 2 and a Munsell 6.2 in Experiment 3. The difference was not significant $[t(18)=0.55, p>.05]$.

In general, our results suggest that the critical geometric factor is relative area, not surroundedness. But is there possibly a residual role for the surround rule over and above the area effect? This is suggested by the relatively high lightness value obtained for the Ganzfeld background in Experiment 2. However, there is another comparison that casts doubt on this possibility. We can compare the unevenly split condition of Experiment 1 with the small disk condition of Experiment 2. In both cases, the lighter area is the smaller area and the darker area is the larger, but, in the small disk condition, the darker area surrounds the lighter, whereas, in the unevenly split condition, it does not. If there were a lightening effect in a surround in addition to that of its area, we would expect the darker region to appear lighter in the small disk condition than in the unevenly split condition. In fact, it appears darker (Munsell 6.9) in the small disk condition than in the unevenly split condition (Munsell 7.8). Nor can this difference, which is significant $[t(18)=3.1, p>.05]$, be attributed to area, because, if anything, the area of the darker region must be greater in the small disk condition.

The crucial variable appears to be the relative area of a region, not whether it surrounds another region. The effect of area on lightness is seen most clearly in the darker of the two regions. In Experiment 1, with a vertical edge, the lightness of the darker region moved up the lightness scale 3.2 Munsell units as its area was expanded to fill most of the visual field. Thus, its lightness moved more than halfway to white, from its middle gray appearance when the two areas were equal. Again, in Experiment 3, when the darker region was central, expanding the area from a small disk to a large oval moved its perceived lightness about half the distance to white, from a perceived reflectance of $13 \%$ (Munsell 4.15 ) to a perceived reflectance of $32 \%$ (Munsell 6.2).

The same trend was found in Experiment 2, even more so when these results are combined with others (our pilot studies and Gilchrist \& Bonato, 1995), showing very light values for the Ganzfeld background in the small disk condition. Experiment 2 also raised interesting questions about the definition of area. The darker region appeared substantially lighter (6.0) when it was the background for the large oval in Experiment 2 than it appeared as a split half (4.5) in Experiment 1, even though it was nominally smaller in Experiment 2 . But, in fact, to ensure that the boundaries of the large oval fell clearly within the visual field, for different observers as well, it was not practical to push the boundaries of the oval out to the very extremes of the visual field. Thus, the area of the Ganzfeld background was not as small as we might have preferred.

But more importantly, the true functional area of this region is indeterminate, in several ways. The visual field has no clearly demarcated boundaries. And even if one were to determine the boundaries of the visual field using the perimeter method, this may not give a valid measure of the phenomenal size of the field. It is well known that surfaces do not appear to stop at the boundaries of the visual field. Nor do they appear to stop at the boundary of a superimposed figure. Thus, it may be that some part of the background that appears to continue behind the figure is included in the computation of background area. Bonato and Cataliotti (1996) showed that a figural region comes to appear self-luminous at a lower luminance value than a background region of equal retinal area, presumably because the perceived area (including the amodal area) of the background region is larger. 
Thus, it may be that the functional size of the Ganzfeld surround, even in the large oval condition, was larger than half of the visual field.

These complications in the determination of area make it difficult to develop a mathematical account of the area effect. A related problem is that area was confounded with retinal eccentricity in our experiments. Experiments are currently underway testing the area effect using pieshaped stimuli. In these domes, the boundary between the lighter and the darker region always consists of two straight lines that radiate out from the center of the dome. This not only gives a convenient fixation point but also equates the proportion of foveal and peripheral area covered by each region. It makes sense to defer further quantification of the area effect until these experiments are completed. We will also distinguish effects that have been confounded in the present experiments, such as overall luminance and area.

Although surroundedness per se does not appear to be crucial, the area principle we obtained may be describable in terms of figure and background, to the extent that figure and background are functions of relative area. Goldhammer (1934), Oyama (1950), and Künnapas (1957) have reported that regions of larger visual area are more likely to be seen as ground.

These findings bring to mind Helson's (1964, p. 292) comment that "we need assume only that within certain limits area acts like luminance, that is, increase in area has the same effect as increase in luminance." It is interesting to recall that Noguchi and Masuda (1971) and Kozaki (1973) reported that the perception of illumination depends on the largest area in the field and on the highest luminance.

As for anchoring of lightness values, the matter can now be summarized quite simply. For simple frameworks, anchoring depends on two factors: relative luminance and relative area. More specifically, the rule for relative luminance, as we have noted, is that the highest of two luminances tends to appear white. The rule for relative area seems to be that the greater of the two areas tends to appear white. The interesting question, of course, is how do relative luminance and relative area combine to anchor surface lightness? In many situations, such as the familiar disk/annulus pattern, the highest luminance (the annulus) also has the greatest area. In these cases the story is quite simple. The region with the high luminance and large area appears white, while the perceived lightness of any darker region depends simply on the ratio of its luminance to that of the maximum, according to Wallach's ratio principle.

When there is a conflict between the highest luminance and the greatest area, the story becomes more complicated. Nevertheless, we have been able to formulate a rule that appears to govern such conditions. We have dubbed it the area rule, and it applies when the highest luminance and the largest area conflict-namely, when the darker of two regions has the larger area. When the area rule applies, an increase in the area of the darker region, at the expense of the area of the lighter region, causes the darker region to appear whiter (the larger the lighter) and the lighter region to begin to move toward a self-luminous appearance. As the darker region approaches $100 \%$ of the area, its lightness approaches white. As the lighter region becomes smaller, it first acquires an enhanced white appearance, and it finally becomes self-luminous.

Although this rule is meant to apply to the study of visual fields that contain only two luminance values, we suppose that some variant of the rule applies to more articulated frameworks and to simple frameworks that are embedded in more complex visual fields. But our claim that area effects begin to emerge only when the darker region is larger than the lighter region should be reserved only for the kind of simple two-part visual fields such as we created with the domes technique. Typically, the kind of display that has been considered simple in this field consists of two relatively bright regions surrounded by darkness or near darkness. Under these conditions of stimulation, there are other reasons to expect a relatively weak area effect even when the lighter region is larger than the darker. But, according to several reports (Gilchrist \& Cataliotti, 1994; Gilchrist et al., in press), that area effect, closely related to Katz's (1935) law of field size, would depend on the total area of the test and inducing regions together, relative to the entire visual field, not on the relative area of test and inducing regions per se.

This rule would have been difficult to derive exclusively from our domes experiments. In the event, the rule emerged as we compared our data with the results of other experiments that have dealt with the role of area in lightness and/or brightness. At the same time, no such rule was identified in any of these previous studies, and it would have been difficult to uncover the rule without the anchoring approach that we have explicitly taken.

There are about a dozen experimental reports in the literature dealing with the dependence of lightness or brightness on some measure of visual area. Many of these were studies of brightness, rather than lightness, and, for many, the display consisted of three regions: two regions surrounded by darkness. In general, our rule appears to account very well for the data. But these reports are split as to whether an area effect occurs when the lighter region is larger than the darker region, some reports showing a weak area effect and some showing none.

Wallach's (1948) experiments were done, in general, with disk/annulus displays in a dark environment. The area of the annulus was about four times that of the disk. He tested the effect of reducing the area of the annulus, and he reported that reducing the annulus to an area roughly equal that of the disk has no effect. But reducing the annulus to an area only one fourth that of the disk has a pronounced effect: the disk (which is the darker region) appears lighter than it otherwise would. This is consistent with our results and with the area rule. As Wallach (1948) observed: "It seems that, once the ring 

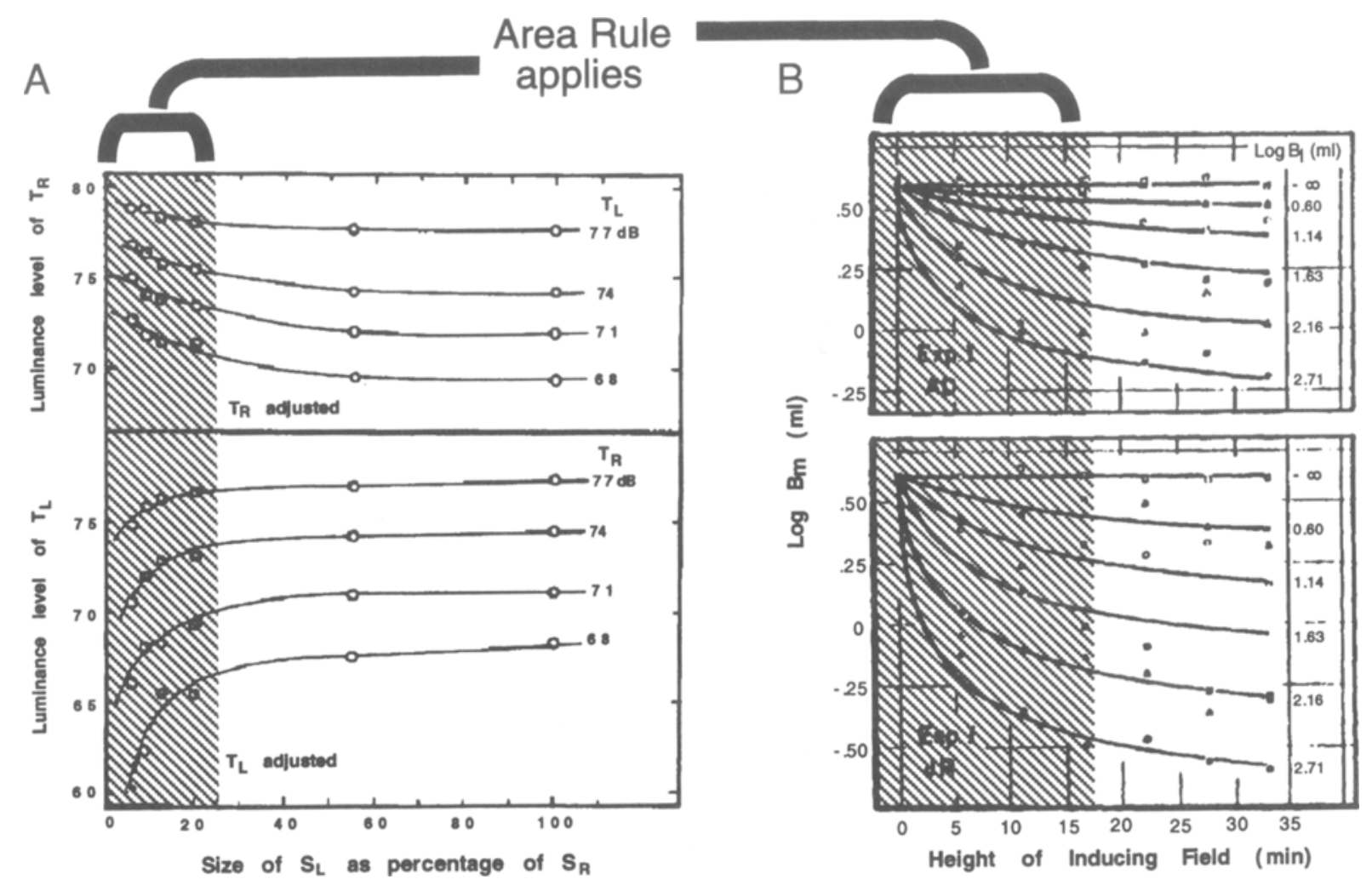

Figure 6. The results of (A) Stevens (1967) and (B) Diamond (1955) showing zones to which the area rule applies. Panel A: From "Brightness Inhibition re Size of Surround," by J. C. Stevens, 1967, Perception \& Psychophysics, 2, p. 191 . Copyright 1967 by the Psychonomic Society, Inc. Panel B: From “Foveal Simultaneous Contrast as a Function of Inducing Field Area," by A. L. Diamond, 1955, Journal of Experimental Psychology, 50, p. 149. Copyright 1955 by The American Psychological Association. Reprinted with permission.

has an area equal to that of the disk, any further increase in its width does not affect the resulting color of the disk" (p. 323).

Stevens (1967) made a parametric test of the effect of variations in the area of the annulus on the brightness of the disk, both within a dark environment. In all of his stimuli, the annulus was the brighter region; the disk was a decrement. Variations in annulus area were shown to be very effective for stimuli (the majority of those tested by Stevens) in which the area of the annulus was less than that of the disk (shown as the shaded area in Figure 6A). For the few cases in which he tested annuli with areas greater than those of the disks, little or no effect was found for annulus area.

Diamond (1955) presented observers with adjacent luminous rectangular test and inducing fields in a dark surround, in order to see how the brightness of the test field varies as a function of the area of the inducing field, which was always brighter than the test field. He found that increasing the size of the inducing field, up to an asymptotic value, darkens the appearance of the test field. Almost all the effect occurred as the inducing field was increased from an area of zero to an area equal to or a bit greater than that of the test field. Beyond this value, the brightness of the test field remained quite steady, close to its asymptotic value. This can be seen in Figure 6B, in which the shaded area represents the zone to which the area rule applies.

In both the Stevens (1967) plot and the Diamond (1955) plot, the $x$-axis represents relative area and the $y$-axis represents a measure of brightness.

Diamond (1962) later reported an experiment in which variations in the area of the test (darker) field did not affect its perceived lightness. But, in this experiment, the test and inducing fields were not adjacent, and the separation between them covaried with the area of the test field. Although this confound prevents an unambiguous interpretation of the data, it seems likely that the lightening of the darker (test) field as its area began to exceed that of the inducing field, as shown in Diamond's (1955) earlier study, was nullified by the decreasing separation between them, known to produce the opposite effect (Fry \& Alpern, 1953; Leibowitz, Mote, \& Thurlow, 1953).

The same difficulty applies to a report by Wallach and his associates (Whipple, Wallach, \& Marshall, 1988). They reported a parametric study of the role of annulus area in a disk/annulus display. Unfortunately, for our purposes, the disk and annulus were never adjacent in their study.

Newson (1958) presented a center/surround display within a beam of light, as in the Gelb (1929) effect. Hold- 
ing constant the luminances of both the darker center square and the lighter annular surrounding region, Newson tested perception of the center square while he varied the area of the surround from zero to an area roughly equal to that of the center square. This range is just the range within which the area rule applies. He obtained a pronounced effect on the lightness of the square. Moreover, his curve (Newson, 1958, Figure 4, p. 94) reached an asymptote just where the areas of the center and surround became equal, suggesting that additional increases in the area of the surround would have no further effect on the lightness of the center.

Stewart (1959), working with a variation of the Gelb effect, presented observers with a large disk that appeared white because it was illuminated by a special beam of light. He measured how much this disk appeared to darken when a smaller white disk was placed on it. All of Stewart's stimuli fell within the zone to which the area rule applies. He found that as the large, and darker, disk became larger relative to the smaller white disk, it also appeared lighter, just as described by the area rule.

Kozaki (1963) tested brightness using a haploscopic technique and a square center/surround display embedded in darkness. The area of the surround was always greater than the area of the test field. She obtained an area effect like that which we obtained ${ }^{3}$ when her test field was an increment, consistent with our area rule. But she also obtained a weak area effect when the test field was a decrement.

Burgh and Grindley (1962) found no effect of surround size in the traditional simultaneous contrast display (gray squares on adjacent black and white backgrounds), even though the area rule should apply to the half of the display containing the black background. But they simply varied the overall size of the display, target squares as well as backgrounds. Thus, although they varied the absolute size of the background regions, they did not vary the relative area between the target and the background. Thus, there is no contradiction here: relative area is the crucial factor, not absolute area. This is not without significance. Absolute area should be an important factor under the conventional lateral inhibition account of brightness (Fry \& Alpern, 1953; Leibowitz et al., 1953).

Yund and Armington (1975) also tested the dependence, in a disk/annulus display, of brightness on relative area. They used their data to compare four spatial variables: width of surround, area of surround, ratio of surround area to center area, and distance between center and surround edges. They concluded that their data were best fit by the metric of distance between center and surround edges, with surround-to-center area ratio a close second. There are several problems with the Yund and Armington study, but the most important one is this: Although they set out to resolve the confusion in previous studies (Diamond, 1955; Heinemann, 1972; Stevens, 1967; Wallach, 1948), Yund and Armington tested the effect of the darker region on the brighter, measuring the perceived brightness of the brighter region, whereas all of the other studies tested the effect of the brighter region on the darker, measuring the brightness of the darker of the two regions. As has been amply demonstrated, the darker region has little or no effect on the perception of the brighter region. The term induction is generally understood to refer to the effect of a brighter region on a darker region. Indeed, when Yund and Armington varied the relative area of the disk and annulus by a factor of 742 , they were able to produce a change in perceived brightness by a factor of less than 2. A twofold effect is negligible compared with the pronounced effects that are obtained when the brightness of the darker region is measured. Given that they studied an effect that is generally regarded as negligible, it is not surprising that Yund and Armington $(1975$, p. 920$)$ referred to "large session-to-session variability" in their data. It might be added that Burgh and Grindley (1962) obtained no effect at all by varying the distance between center and surround edges.

In a well-known study, Heinemann (1955) studied the perception of a disk of constant luminance as the luminance of a surrounding annulus was increased from zero to values higher than that of the disk. In general, he found that variations in the luminance of the annulus have a marked effect on the appearance of the disk only when annulus luminance exceeds disk luminance-in other words, only when the highest luminance changes (see Figure 7). This basic pattern invites an anchoring approach. Consider the pattern of results that are predicted if Heinemann's experiment is analyzed from an anchoring perspective, using the highest luminance rule. The data fall along a horizontal line until the increment/ decrement threshold is crossed, at which point the data fall into a slope of -1 , as shown in Figure 7C. A comparison between this ideal pattern and Heinemann's data reveals three discrepancies: (1) The second half of Heinemann's curve drops much faster than a slope of -1 . (2) The perceived brightness of the disk begins to plummet, even before annulus luminance exceeds disk luminance (at the increment/decrement threshold). We will refer to this as the breakpoint offset. (3) Before the disk's brightness begins to plummet, while its luminance is still an increment, increases in annulus luminance cause a small enhancement of the perceived brightness of the disk.

The first of these discrepancies can be explained by Heinemann's use of a comparison disk surrounded by darkness. When the comparison disk has its own annulus (and thus appears as a surface color), the -1 slope is obtained (see Arend \& Goldstein, 1987; Gilchrist \& Bonato, 1995; Heinemann, 1955, Experiment 2; Wallach, 1948).

As for the second and third discrepancies, Diamond (1960) and Heinemann and Chase (1995) have offered mathematical accounts of these effects. However, neither of these models can account for all of the following features of the empirical data: (1) Both the enhancement effect and the breakpoint offset appear under some conditions (Heinemann, 1955; Torii \& Uemura, 1965), but not others (Diamond, 1953; Horeman, 1963; Leibowitz 

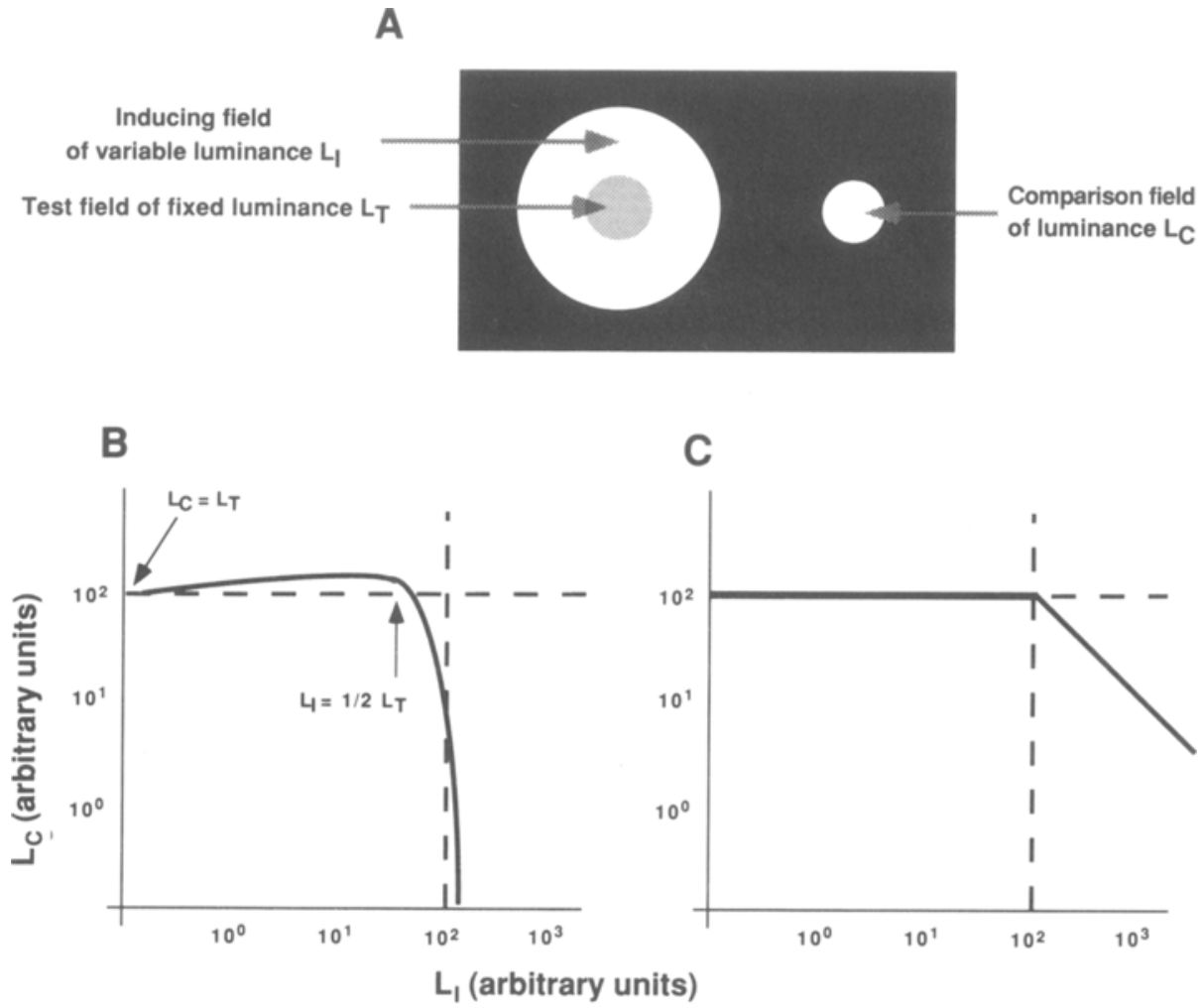

Heinemann data

Predicted by HLR

Figure 7. The configuration (A) and results (B) from Heinemann (1955), with the results (C) as predicted solely by the highest luminance rule.

et al., 1953; Torii \& Uemura, 1965). (2) The two effects appear and disappear together. (3) Both of these effects occur when the annulus is darker than the disk, but only when the area of the annulus is greater than the area of the disk (Diamond, 1960; Heinemann, 1972; Torii \& Uemura, 1965). These are exactly the conditions under which the area rule applies.

We believe these two effects are the product of anchoring based on relative area, which overlays the basic pattern of anchoring based on the highest luminance. In the absence of an area effect, anchoring is governed simply by the highest luminance rule. In that case, the annulus comes to appear white only when its luminance surpasses that of the disk. But when the annulus is perceptually lightened by enlarging its area, it can come to appear white even before it surpasses the disk in luminance. As soon as the annulus comes to appear white, it becomes the anchor; whenever the luminance of the anchor increases, all other surfaces in the framework are darkened. Here, the only other surface is the disk. Because the annulus becomes the anchor before it crosses the increment/ decrement threshold, the disk must also begin to darken before the increment/decrement threshold is reached. The breakpoint offset thus follows from the area effect.
We believe that the enhancement effect is also a logical consequence of the area rule. The large area of the annulus gives it a higher lightness value, according to the rule, even with no change in luminance values. In turn, this lightening of the annulus creates a discrepancy between the actual disk/annulus stimulus difference and the perceived disk/annulus stimulus difference. The discrepancy shows up in visual experience in two ways. First, there is a compression in the mapping from stimulus range to perceptual grayscale range. Second, the higher luminance region appears not merely white but superwhite. Perceiving an enhanced brightness in the disk reduces this contradiction.

Apparently, when a disk/Ganzfeld configuration is used instead of a disk/annulus, the discrepancy is absorbed in a different way. Schouten and Blommaert (1995a, 1995b) have reported a phenomenon that they describe as a novel compression mechanism in the luminance-brightness mapping. They call it brightness indention. Using a display that consisted of two disks within a Ganzfeld, Schouten and Blommaert found that when both disks are brighter than the Ganzfeld, the Ganzfeld background does not appear homogeneous: It appears darker in the immediate vicinity of the disks, creating a kind of dark 
halo around each. The phenomenon occurs only in the zone to which our area rule applies, when the Ganzfeld, with its large area, is darker than both of the disks. As with the disk/annulus case, we can speak of a competition between the tendency for the surround to appear white because it has the greatest area and the tendency for a disk to appear white because it has the highest luminance. Apparently, in the Ganzfeld case, the conflict is resolved by sacrificing the perceived homogeneity of the Ganzfeld. Newson (1958, p. 87) described the same phenomenon in his Gelb effect experiments.

The surround rule that was proposed by Gilchrist and Bonato (1995) was designed to resolve the contradiction between the highest luminance rule and the phenomenon of luminosity. If the surround rule is not valid, what about the luminosity problem? Under the conditions to which the area rule applies - that is, when the darker of the two regions has the greater relative area-an increase in the relative area of the darker region (which is equivalent to a decrease in the area of the lighter region) appears to push the lighter region toward a luminous appearance.

There seems to be a lag here, however, that does not occur for the darker region. The effect is best explained by considering what happens to the appearance of the lighter region as the area of the darker region grows at the expense of the lighter region, with no change in luminance values. We will assume a light:dark luminance ratio that is about half of the black: white ratio (say, 5:1). It will be helpful to refer to Figure 8 at this point. As the relative area of the darker region increases to well beyond $50 \%$, no change occurs in the matched lightness of the lighter region. This is the compression of which we spoke earlier. Because the darker region appears to lighten while the lighter region continues to appear white, the perceived lightness difference between them becomes smaller relative to the physical difference between them. As relative area shifts further in favor of the darker region, the lighter region begins to appear as a kind of superwhite. We use the term superwhite because it appears as a very bright white, but not quite self-luminous. Evans (1948, 1959, 1974) has used the term fluorence for such an appearance between white and self-luminosity, and we believe it is the same phenomenon as Heinemann's (1955) enhancement effect.

Finally, when the area of the larger region becomes very large relative to the area of the lighter region, the lighter region is forced into self-luminosity. In fact, we obtained very little empirical evidence on this last point. There was at least a trend in Experiment 1. Experiment 3 was not relevant because the conditions for self-luminosity were not present. We obtained no reports of luminosity in Experiment 2, contrary to the implications in Figure 8. But, as noted earlier, our Experiment 2 results were not entirely consistent with other similar experiments, especially with regard to self-luminosity. Self-luminosity reports were obtained from $60 \%$ of the observers in a very similar condition run by Gilchrist and Bonato (1995) and from $50 \%$ of the observers in an earlier pilot study we ran. Finally, if the lightness of the darker region approaches white as its area approaches $100 \%$ (a conclusion strongly supported by our results), the only logical outcome for the lighter region is self-luminosity.

The phenomenon of self-luminosity is a problem when anchoring is considered only in terms of relative lumi-

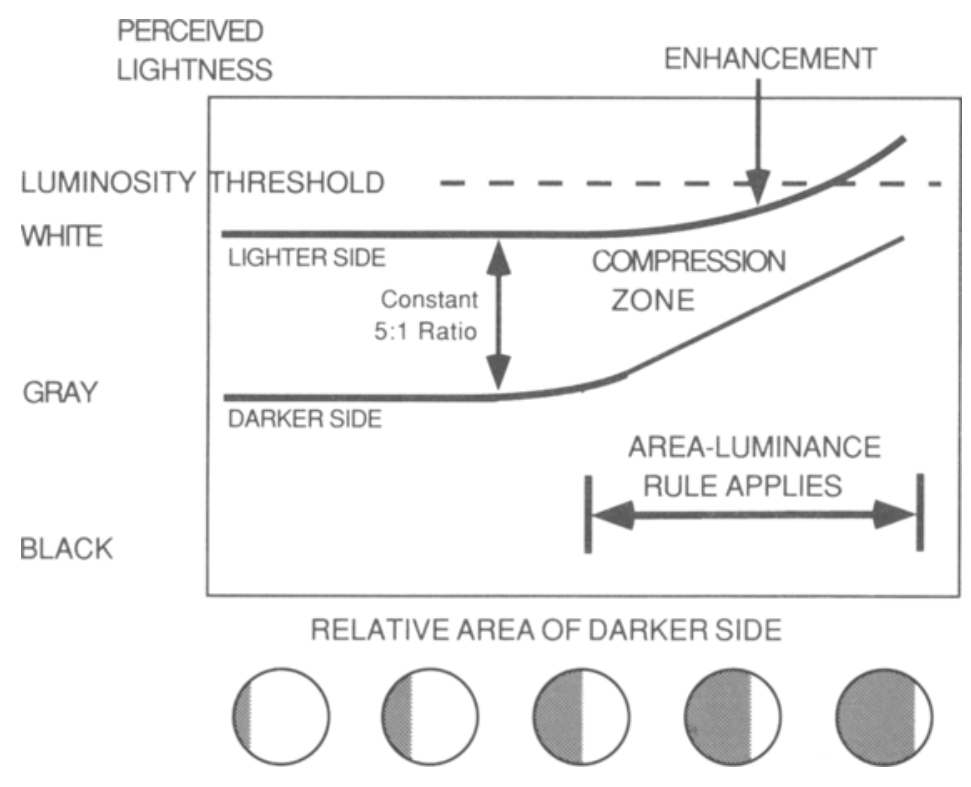

Figure 8. Schematic summarizing the appearance of two regions whose luminance values stand in a constant ratio of 5:1, as a function of the area of the darker region. 
nance, specifically in terms of the following rule: highest luminance $=$ white. When anchoring is considered in terms of relative area, the contradiction between selfluminosity and the highest luminance rule can be resolved.

Bonato and Gilchrist (1999) measured the luminosity threshold as a function of the visual area of a target region. They found that when the area of a target was increased by a factor of about 17 , the luminance required to make it appear self-luminous was increased by a factor of about 3 . Thus, for example, at a target:background luminance ratio of $4: 1,90 \%$ of the observers rated the small target as luminous, whereas only $20 \%$ rated the large target as luminous. These effects appear to be completely consistent with the analysis we offer here. The area effects they obtained, as in the present study, occurred when the higher of the two luminances had the smaller relative area, conditions to which the area rule applies.

Bonato and Gilchrist also found, by varying the distance of the display from the observer, that it is perceived area, not retinal area, that is crucial, just as Rock has found for other spatial variables such as proximity (Rock \& Brosgole, 1964) and location (Rock \& Ebenholtz, 1962). In a fascinating twist on the notion of perceived area, Bonato and Cataliotti (1996) have found that a region perceived as ground in a face/vase type of figure does show a higher luminosity threshold than when the same region is seen as figure, without changing retinal area. Ti:is appears to be due to the larger perceived area of the ground, because it appears to extend behind the figural region, as Rubin (1921) noted. But Bonato and Gilchrist found that causing a target region to appear as background simply by placing a smaller figural region on it has no effect on the luminosity threshold, presumably because this has no effect on its perceived area. Using the same technique, Noguchi and Kozaki (1985) found that causing a target to appear as background has no effect on its lightness.

The area rule does not appear to be consistent with the results reported by Helson and his associates (Helson \& Joy, 1962; Helson \& Rohles, 1959) in their studies of lightness assimilation (Bezold, 1874), and we can offer no coherent explanation for this discrepancy.

Strictly speaking, the area rule applies only to simple two-part displays. Its applicability to more complex images remains to be determined. Nevertheless, several steps have already been taken. First, we have seen that the rule works for three-part displays involving two luminous regions presented within a dark surround (Diamond, 1955; Heinemann, 1972; Kozaki, 1963; Newson, 1958; Stevens, 1967; Stewart, 1959; Wallach, 1948; ). Remarkably, the visual system seems able to treat even such ecologically invalid displays as representing real surfaces. Second, it should be noted that the dependence of the luminosity threshold on relative area was found by Bonato and Gilchrist in a highly articulated environment, not under reduced conditions. Gilchrist and Cataliotti (1994) have obtained significant effects of relative area on the lightness of opaque surfaces in highly complex images.
When discussing area effects in more complex images, the work of Katz (1935) is very important. Katz was apparently the first to discuss area effects in lightness perception, and he formulated the first and second laws of field size. He used the term field to refer to a region of common illumination. According to his laws, the larger the field, the greater the degree of lightness constancy. The first law defines size in terms of visual angle and the second defines size in terms of perceived size. Katz believed that both of these laws are separately effective. But apparently Katz failed to recognize that his demonstration $(1935$, p. 215) of the effect of retinal size on lightness constancy was confounded; it allowed perceived size to vary as well. The effects of area on lightness (Gilchrist \& Cataliotti, 1994) and on the luminosity threshold (Bonato \& Gilchrist, 1999) appear to be governed by perceived area, not retinal area.

More work is needed to see whether variations in the size of single regions, as studied in the present experiments, follow the same principles as variations in the size of more complex frameworks and how both relate to Katz's laws of field size.

This work shows the fruitfulness of studying the anchoring problem. It may be that the whole concept of lightness contrast, or lightness induction, can be usefully replaced by the concept of lightness anchoring. Recall that in Heinemann's (1955) classic study of brightness induction, variations in the luminance of an annulus were shown to have a substantial influence on the perceived brightness of the enclosed disk only when the luminance of the annulus exceeds that of the disk. This is exactly what would be expected on the basis of anchoring by the highest luminance. Diamond (1955) and Stevens (1967) fitted curves to their data on the basis of lateral inhibition as an explanatory concept. But, as we can see in Figure 6, it appears that their data really fall into two qualitative regions, defined by the intersection of relative luminance and relative area. Perhaps both effects of relative area and effects of relative luminance in lightness and brightness can be better understood in terms of anchoring than in terms of lateral inhibition.

\section{REFERENCES}

Adelson, E. H., \& Pentland, A. P. (1990). The perception of shading and reflectance (Vision and Modeling Tech. Report 140). Cambridge, MA: MIT Media Laboratory.

AREND, L. (1994). Surface colors, illumination, and surface geometry: Intrinsic-image models of human color perception. In A. Gilchrist (Ed.), Lightness, brightness, and transparency (pp. 159-213). Hillsdale, NJ; ErIbaum.

AREND, L., \& GOLDSTEIN, R. (1987). Simultaneous constancy, lightness and brightness. Journal of the Optical Society of America A, 4, 2281-2285.

BERGSTRÖM, S. S. (1977). Common and relative components of reflected light as information about the illumination, colour, and threedimensional form of objects. Scandanavian Journal of Psychology, 18, 180-186.

BEZOLD, W. von (1876). Die Farbenlehre [The theory of color] (S. R. Koehler, Trans.). Brunswick: Westermann. 
Bonato, F., \& Catalıotti, J. (1996). Perceived area and figure/ground relations affect the luminosity threshold. Investigative Ophthalmology \& Visual Science, 37, s174.

Bonato, F., \& Gilchrist, A. (1999). Perceived area and the luminosity threshold. Perception \& Psychophysics, 61, 786-797.

Brown, R. O. (1994). The world is not gray. Investigative Ophthalmology \& Visual Science, 35, 2165.

BROWN, R. [O.], \& MACLEOD, D. (1992). Saturation and color constancy. Advances in Color Vision Technical Digest, 4, 110-111.

BRUNo, N. (1992). Lightness, equivalent backgrounds, and the spatial integration of luminance. Perception, 21 (Suppl.), 80.

Bruno, N., Bernardis, P., \& Schirillo, J. (1997). Lightness, equivalent backgrounds, and anchoring. Perception \& Psychophysics, 59, 643-654.

BuchsBauM, G. (1980). A spatial processor model for object color perception. Journal of the Franklin Institute, 310, 1-26.

BURGH, P., \& GRINDLEY, G. C. (1962). Size of test patch and simultaneous contrast. Quarterly Journal of Experimental Psychology, 14, 89-93.

CAtaliotTI, J., \& Gilchrist, A. [L.] (1995). Local and global processes in surface lightness perception. Perception \& Psychophysics, 57, 125-135.

Cornsweet, T. N. (1970). Visual perception. New York: Academic Press.

Diamond, A. L. (1953). Foveal simultaneous brightness contrast as a function of inducing- and test-field luminances. Journal of Experimental Psychology, 45, 304-314.

DiAMOND, A. L. (1955). Foveal simultaneous contrast as a function of inducing field area. Journal of Experimental Psychology, 50, 144-152.

DiAmOND, A. L. (1960). A theory of depression and enhancement in the brightness response. Psychological Review, 67, 168-199.

Diamond, A. L. (1962). Simultaneous contrast as a function of testfield area. Journal of Experimental Psychology, 64, 336-346.

EvaNS, R. M. (1948). An introduction to color. New York: Wiley.

EvaNs, R. [M.] (1959). Fluorescence and gray content of surface colors. Journal of the Optical Society of America, 49, 1049.

Evans, R. M. (1974). The perception of color. New York: Wiley.

FRY, G. A., \& ALPERN, M. (1953). The effect of a peripheral glare source upon the apparent brightness of an object. Journal of the Optical Society of America, 43, 189-195.

GeLB, A. (1929). Die "Farbenkonstanz" der Sehdinge [The "color constancy" of visual objects]. In W. A. von Bethe (Ed.), Handbuch der Normalen und Pathologischen Psychologie (Vol. 12, pp. 594-678). Berlin: Springer-Verlag.

GILCHRIST, A. (1979). The perception of surface blacks and whites. Scientific American, 240, 112-123.

Gilchrist, A., \& Bonato, F. (1995). Anchoring of lightness values in center/surround displays. Journal of Experimental Psychology: Human Perception \& Performance, 21, 1427-1440.

Gilchrist, A., \& CATALIOTTI, J. (1994). Anchoring of surface lightness with multiple illumination levels. Investigative Ophthalmology \& $V i$ sual Science, 35, 2165.

Gilchrist, A., Kossyfidis, C., Bonato, F., Agostini, T., Cataliotti, J., LI, X., SPEhar, B., \& SzURA, J. (in press). A new theory of lightness perception. Psychological Review.

Goldhammer, H. (1934). The influence of area, position, and brightness in the perception of a reversible configuration. American Journal of Psychology, 46, 186-206.

HeINEMANN, E. G. (1955). Simultaneous brightness induction as a function of inducing- and test-field luminances. Journal of Experimental Psychology, 50, 89-96.

HeinemanN, E. G. (1972). Simultaneous brightness induction. In D. Jameson \& L. M. Hurvich (Eds.), Handbook of sensory physiology (Vol. 7, Pt. 4, pp. 147-169). Berlin: Springer-Verlag.

Heinemann, E. G., \& Chase, S. (1995). A quantitative model for simultaneous brightness induction. Vision Research, 35, 2007-2020.

Helson, H. (1964). Adaptation level theory. New York: Harper \& Row.

Helson, H., \& JoY, V. (1962). Domains of lightness, assimilation and contrast effects in vision. Psychologische Beiträge, 6, 405-415.
HELSON, H., \& RoHLEs, F. (1959). A quantitative study of reversal of classic lightness-contrast. American Journal of Psychology, 72, 530-538.

HoREMAN, H. W. (1963). Inductive brightness depression as influenced by configurational conditions. Vision Research, 3, 121-130.

HoRN, B. K. P. (1974). Determining lightness from an image. Computer Graphics \& Image Processing, 3, 277-299.

HURLBERT, A. (1986). Formal connections between lightness algorithms. Journal of the Optical Society of America A, 3, 1684-1693.

JAMESON, D., \& HURVICH, L. M. (1964). Theory of brightness and color contrast in human vision. Vision Research, 4, 135-154.

KaTZ, D. (1935). The world of colour. London: Kegan Paul, Trench, Trubner.

KoZAKI, A. (1963). A further study in the relationship between brightness constancy and contrast. Japanese Psychological Research, 5, 129-136.

KozaKI, A. (1973). Perception of lightness and brightness of achromatic surface color and impression of illumination. Japanese Psychological Research, 15, 194-203.

KüNNAPAS, T. (1957). Experiments on figural dominance. Journal of Experimental Psychology, 53, 31-39.

LAND, E. H., \& MCCANN, J. J. (1971). Lightness and retinex theory. Journal of the Optical Society of America, 61, 1-11.

Leibowitz, H., Mote, F. A., \& ThurLow, W. R. (1953). Simultaneous contrast as a function of separation between test and inducing fields. Journal of Experimental Psychology, 46, 453-456.

Newson, L. J. (1958). Some principles governing changes in the apparent lightness of test surfaces isolated from their normal backgrounds. Quarterly Journal of Experimental Psychology, 10, 82:-95.

Noguchi, K., \& KozaKI, A. (1985). Perceptual scission of surfacelightness and illumination: An examination of the Gelb effect. Psychological Research, 47, 19-25.

NoguCHI, K., \& MASUda, N. (1971). Brightness changes in a complex field with changing illumination: A re-examination of Jameson and Hurvich's study of brightness constancy. Japanese Psychological Research, 13, 60-69.

OYAMA, T. (1950). Figure-ground dominance as a function of sector angle, brightness, hue, and orientation. Journal of Experimental Psychology, 60, 299-305.

RoCK, I., \& BROSGOLE, L. (1964). Grouping based on phenomenal proximity. Journal of Experimental Psychology, 67, 531-538.

RoCk, I., \& EbENHoltz, S. (1962). Stroboscopic movement based on change of phenomenal rather than retinal location. American Journal of Psychology, 75, 193-207.

RuBIN, E. (1921). Visuell wahrgenomenne Figuren [Visually perceived figures]. Copenhagen: Gyldendal.

SCHIRILLO, J., \& SHEVELL, S. (1993). Lightness and brightness judgments of coplanar retinally noncontiguous surfaces. Journal of the Optical Society of America A, 12, 2442-2452.

Schouten, G., \& Blommaert, F. J. J. (1995a). Brightness constancy in a Ganzfeld environment. Perception \& Psychophysics, 57, 1012-1022.

Schouten, G., \& Blommaert, F. J. J. (1995b). Brightness indention: A novel compression mechanism in the luminance-brightness mapping. Perception \& Psychophysics, 57, 1023-1031.

Stevens, J. C. (1967). Brightness inhibition re size of surround. Perception \& Psychophysics, 2, 189-192.

Stewart, E. (1959). The Gelb effect. Journal of Experimental Psychology, 57, 235-242.

TORII, S., \& UEMURA, Y. (1965). Effects of inducing luminance and area upon the apparent brightness of a test field. Japanese Psychological Research, 2, 86-100.

WALLACH, H. (1948). Brightness constancy and the nature of achromatic colors. Journal of Experimental Psychology, 38, 310-324.

WaLlaCh, H. (1976). On perception. New York: Quadrangle/The New York Times Book Co.

Whipple, W. R., Wallach, H., \& Marshall, F. J. (1988). The effect of area, separation, and dichoptic presentation on the perception of achromatic color. Perception \& Psychophysics, 43, 367-372.

YUND, E. W., \& ARMINGTON, J. C. (1975). Color and brightness contrast effects as a function of spatial variables. Vision Research, 15, 917-929. 


\section{NOTES}

1. Our disk subtended a visual angle of $1.35^{\circ}$; Gilchrist and Bonato's (1995) disk subtended $4.8^{\circ}$

2. Absolute luminance levels were about six times lower in our study than in Gilchrist and Bonato's (1995) study, yet they were about the same as in our pilot study. Our small disk was in fact a paper disk cemented onto the black Ganzfeld background, whereas the disk in Gilchrist and Bonato's experiment was a round aperture that revealed part of a white surface in different illumination. Again, though, a paper disk was used in our pilot study. Our Ganzfeld surround was physically black, whereas that used by Gilchrist and Bonato was physically white. But a black Ganzfeld background was used in the pilot study as well.

3. Although Figure 5 in Kozaki (1963) appears to show an area effect opposite to what we report, Kozaki has confirmed in a personal communication (September, 1995) that this is merely due to the omission of minus signs on the values along the $x$-axis that show "log ratio of test field area to inducing field area."

(Manuscript received March 17, 1997; revision accepted for publication March 18, 1998.) 